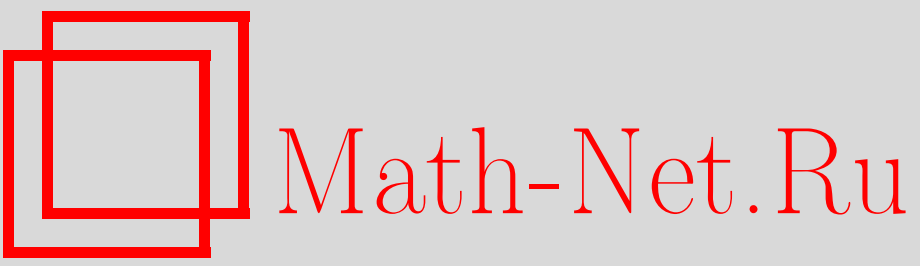

П. А. Писарев, О множестве чисел, представимых в виде цепных дробей с ограниченными неполными частными, УМН, 2000, том 55, выпуск 5, 177-178

DOI: https://doi.org/10.4213/rm327

Использование Общероссийского математического портала Math-Net.Ru подразумевает, что вы прочитали и согласны с пользовательским соглашением

http://www.mathnet.ru/rus/agreement

Параметры загрузки:

IP: 54.174 .149 .18

26 апреля 2023 г., $17: 05: 20$ 


\title{
О МНОЖЕСТВЕ ЧИСЕЛ, ПРЕДСТАВИМЫХ В ВИДЕ ЦЕПНЫХ ДРОБЕЙ С ОГРАНИЧЕННЫМИ НЕПОЛНЫМИ ЧАСТНЫМИ
}

\author{
П. А. ПИСАРЕВ
}

Рассмотрим множество $F(k)$ всех иррациональных чисел отрезка $[0 ; 1]$, у которых все неполные частные разложения в цепную дробь ограничены числом $k \in \mathbb{Z}$. Известно [1], [2; Ch. 4], что его можно получить стандартной процедурой выкидьвания счетного числа непересекающихся интервалов: $F(k)=\mathscr{I} \backslash \bigcup_{\nu} \Delta_{\nu}$ с выполнением $\tau$-свойства: каждьй раз интервал $\Delta_{\nu}$ выкидывается из некоторого отрезка $M_{\nu} \subset \mathscr{I}$, разбивая его таким образом, что $M_{\nu}=N_{1} \sqcup \Delta_{\nu} \sqcup N_{2}$ и $\min \left\{\left|N_{1}\right|,\left|N_{2}\right|\right\} \geqslant \tau\left|\Delta_{\nu}\right|$. (Через $|\cdot|$ здесь и в дальнейшем обозначается длина отрезка или интервала.) Например, в [3] утверждается, что при $k \geqslant 4$ выполняется $\tau \geqslant k / 4$, а достаточно точные значения $\tau$ при $k=2,3$ использовались в [4]. Однако детального описания, как именно выполняется $\tau$-свойство, мы нигде не встречали. В связи с этим в настоящей заметке мы описьваем структуру выполнения $\tau$-свойства для $F(k)$.

Итак, рассмотрим множество чисел, у которых первые $r$ неполных частных ограничены величиной $k$ и фиксированы, оно представляет собой некоторый отрезок $M$. Из него требуется удалить $k-1$ интервал, состоящий из чисел, неполные частные которых превышают $k$. Введем обозначение $\left[*, i_{1}, i_{2}, \ldots\right]=\left[0 ; a_{1}, a_{2}, \ldots, a_{r}, i_{1}, i_{2}, \ldots\right]$, где все $a_{j} \leqslant k \forall j \leqslant r, \frac{p_{r}}{q_{r}}=\left[0 ; a_{1}, \ldots, a_{r}\right]$ - подходящая дробь $n$-го порядка. Пусть

$$
\xi:=\min F(k)=[0 ; \overline{k, 1}], \quad \max F(k)=[0 ; \overline{1, k}]=\frac{1}{1+\xi}=\frac{1}{\xi}-k .
$$

Разбиение исследуемого отрезка будет осуществляться последовательно $k-1$ шагом. На $i$-м шаге отрезок $M_{i}$, с концами $[*, \overline{k, 1}]$ и $[*, \overline{1, k}]$, будет дробиться следующим образом: $M_{i}=N_{i}^{1} \sqcup \Delta_{i} \sqcup N_{i}^{2}$, где концы выкидываемого интервала $\Delta_{i}$ суть $[*, i+1, \overline{k, 1}]$ и $[*, i, \overline{1, k}]$.

Длины $\left|N_{i}^{1}\right|,\left|\Delta_{i}\right|,\left|N_{i}^{2}\right|$ легко находятся ([5] или [1]). Пусть $t=\frac{q_{r}}{q_{r-1}}$. Тогда

$$
\begin{gathered}
\left|\Delta_{i}\right|=\frac{\xi-1 / \xi+k+1}{(t(\xi+i+1)+1)(t(1 / \xi-k+i)+1) q_{r-1}^{2}}, \\
\left|N_{i}^{1}\right|=\frac{1 / \xi-\xi-i-1}{(t / \xi+1)(t(\xi+i+1)+1) q_{r-1}^{2}}, \quad\left|N_{i}^{2}\right|=\frac{1 / \xi-k-\xi}{(t(1 / \xi+i-k)+1)(t(i+\xi)+1) q_{r-1}^{2}} .
\end{gathered}
$$

Ниже в утверждениях 1-3 отрезок $M$, из которого мы выкидываем $k-1$ интервал, соответствующий следующему этажу цепной дроби, считаеся зафиксированным.

УтвеРЖДЕНИЕ 1. $\frac{\Delta_{i+1}}{N_{i+1}^{2}}>\frac{\Delta_{i}}{N_{i}^{2}} \quad \forall i=\overline{1, k-2} ; \quad \frac{\Delta_{k-1}}{N_{k-1}^{2}}<\frac{\Delta_{k-1}}{N_{k-1}^{1}}$.

Далее рассмотрим величину $\omega_{i}=\Delta_{i} / N_{i}^{1}, i=\overline{1, k-1}$.

УТвеРЖДЕНИЕ 2. 1) Для фиксированного отрезка $M$ возможны только два случая:

(i) величина $\omega_{i}$ с ростом $i$ монотонно возрастает;

(ii) величина $\omega_{i}$ при увеличении $i$ сначала убъвает, затем возрастает, причем минимальное значение она принимает в точке $\frac{k}{2}-1$, если $k$-четное, или в одной из двух точек $\frac{k \pm 1}{2}-1$, если $k$ - нечетное.

2) При $k=3,4$ выполняется только случай (i), а отрезков $M$, для которых выполнено свойство (ii), нет.

3) При $k=5$ в процессе построения множества $F(5)$ встречаются отрезки $M$ как с условием (i), так и с условием (ii), причем и тех и других бесконечное число.

4) При $k \geqslant 6$ в расматриваемой прочедуре построения множества $F(k)$ возникают только отрезки $M$ со свойством (ii), а отрезков со свойством (i) нет.

Чтобы наметить доказательство утверждения 2, положим

$$
\omega_{i+1}-\omega_{i}=A_{i} \cdot(2 i+2+\xi+1 / t-k), \quad A_{i}>0 .
$$


Утверждение 2 выводится из перечисленных ниже наблюдений а)-в).

a) При $k=3$ или 4 всегда выполняется $2 i+2+\xi+\varepsilon-k>0$ и, следовательно, $\omega_{i}$ возрастает.

б) При $k=5$ все определяется рассмотрением $i=1$. Для отрезков $M$ типа (i) необходимо выполнение неравенства $\xi+\varepsilon>1$. Так как $\xi=(3 \sqrt{5}-5) / 10$, то это равносильно $\left[a_{r} ; a_{r-1}, \ldots, a_{1}\right]$ $<(5+\sqrt{5}) / 6$. Но это неравенство будет выполняться для каждой подходящей дроби числа $[1 ; \overline{5,1}]$. Значит, отрезков $M$, для которых выполнено (i), найдется бесконечное число. Аналогично устанавливается неограниченность числа отрезков $M$, для которых имеет место (ii).

в) При $k \geqslant 6$, полагая $i=1$, видим, что $2+2+\xi+\varepsilon-k<0$. Значит, $\omega_{i}$ сперва убывает, а затем при больших $i$ возрастает. Ясно, как определить точное значение $i$, где убывание сменяется возрастанием.

УТВЕРЖДЕНИЕ 3. $\omega_{k-1}>\omega_{1}$.

Из утверждений 1-3 вытекает

СледСтвиЕ. $F(k)$ яляется $\tau(k)$-множеством, где $\tau(k)=\inf _{M} \frac{1}{\omega_{i}}=\min _{M} \frac{N_{k-1}^{1}}{\Delta_{k-1}}$, причем это значение $\tau(k)$ является максимально возможным и будет достигнуто уже на первом шаге дробления отрезка $\mathscr{I}$, а именно при $r=0$.

ДокАЗАТЕльство. В работе [6] было установлено, что если все $\Delta_{\nu}$ выбрасьваются с условием $\Delta_{\nu} \geqslant \Delta_{\nu+1}$, то изменение порядка их выкидывания не сможет уменьшить первоначально полученное значение $\tau$. Имеют место следующие два факта:

1) $\forall M \Delta_{i} \geqslant \Delta_{i+1}, i=\overline{1, k-1}$

2) $\forall M \Delta_{i} \geqslant N_{i+1}^{1}, i=\overline{1, k-2}$,

вытекающие из формул для длин $\Delta_{i}$ и $N_{i}^{1}$. Это позволяет построить алгоритм выбрасывания $\Delta_{\nu}$ по невозрастанию длин с сохранением значения $\tau(k)$.

Из последнего следствия легко получается асимптотическое равенство.

ПредЛОЖениЕ. $\tau(k)=\frac{k}{2}-\frac{3}{4}+O\left(\frac{1}{k}\right), \quad k \rightarrow \infty$.

Некоторые вычисленные нами значения $\tau(k)$ при малых $k$ помещены в следующей таблице. Похожие результаты были получены в [7].

\begin{tabular}{|c|c|c|c|c|c|c|c|c|c|}
\hline$k$ & 2 & 3 & 4 & 5 & 6 & 7 & 10 & 20 & 50 \\
\hline$\tau(k) \approx$ & 0.366 & 0.822 & 1.300 & 1.789 & 2.281 & 2.776 & 4.267 & 9.257 & 24.253 \\
\hline
\end{tabular}

\section{СПИСОК ЛИТЕРАТУРЫ}

[1] Hall M. Jr. // Ann. of Math. (2). 1947. V. 48. P. 966-993. [2] Cusick T. W., Flachive M.E. The Markoff and Lagrange spectra. Providence, RI: Amer. Math. Soc., 1989. [3] Мощевитин Н. Г. // УМН. 1997. Т. 52. №6. С. 145-146. [4] Cusick T. W. // Proc. Amer. Math. Soc. 1973. V. 38. № 2. Р. 253-254. [5] Хинчин А.Я. Цепные дроби. М.: Наука, 1978. [6] Williams R. F. // Contemp. Math. 1991. V. 117. P. 163-175. [7] Astels S. // Trans. Amer. Math. Soc. 1999. V. 352. № 1. P. 133-170. 